\title{
eJRIEPS
}

Ejournal de la recherche sur l'intervention en éducation physique et sport

$21 \mid 2010$

Varia

\section{La couleur des maillots en sport collectif : chroniques et légendes contées au peuple des supporters}

Jean-Francis Gréhaigne et Marie-Paule Poggi

\section{(2) OpenEdition}

Édition électronique

URL : http://journals.openedition.org/ejrieps/4736

ISSN : 2105-0821

Éditeur

ELLIADD

Référence électronique

Jean-Francis Gréhaigne et Marie-Paule Poggi, « La couleur des maillots en sport collectif : chroniques et légendes contées au peuple des supporters », eJRIEPS [En ligne], 21 | 2010, mis en ligne le 01 juillet 2010, consulté le 27 février 2020. URL : http://journals.openedition.org/ejrieps/4736

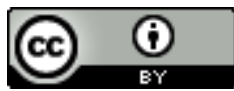

La revue eJRIEPS est mise à disposition selon les termes de la Creative Commons Attribution 4.0 International License. 
La couleur des maillots en sport collectif : chroniques et légendes contées au peuple des supporters

Ce texte, en signe de clin d'œil, constitue un passage de témoin entre l'éditeur scientifique sortant et l'éditrice scientifique entrante ...

Jean-Francis Gréhaigne \& Marie-Paule Poggi

GRIAPS, Université de Franche-Comté. Besançon. France

Résumé

À première vue, les couleurs brillantes portées par les joueurs de la "Tribu du football » ont l'air d'être simplement décoratives, mais cela est une erreur. Pour un club ou une équipe nationale, le maillot est plus qu'un simple vêtement, il est également et surtout le symbole de l'histoire et de l'héritage d'une équipe, d'un club, parfois, d'une ville entière. C'est vrai, ces couleurs constituent une touche attrayante, chatoyante au-dessus du vert du gazon mais, en fait, l'intérêt visuel n'est pas la fonction première de ces couleurs. En réalité, elles sont des signaux essentiels pour la tribu. Comme les couleurs exhibées par beaucoup d'animaux ou de fleurs, elles envoient des messages essentiels à propos de ceux qui les portent. Ainsi, s'intéresser aux histoires et légendes des maillots pourrait au premier abord sembler anecdotique, voire triviale, peu importe après tout que les joueurs endossent des maillots rayés ou unis, rouges ou verts. Ce serait toutefois oublier leur fonction emblématique et identitaire. Avec eux, le corps se fait signe et se charge de sens. II devient le support de représentations et de pratiques particulières qui permettent de délimiter des territoires et de se différencier. Finalement, le maillot exacerbe la puissance sémique du corps.

L'impact et l'utilisation de la couleur dans des contextes sportifs reste un domaine de recherche relativement peu exploré. Attrill, Gresty, Hill et Barton (2005) ont montré que lorsque les tenues rouges et bleues ont été assignées au hasard à des participants de combat de boxe olympique, de taekwondo, de lutte libre ou gréco-romaine, la fréquence des gagnants portant du rouge a été significativement plus élevée qu'une simple répartition due au hasard. Ainsi, la couleur des vêtements de sport a montré qu'elle influence le résultat des rencontres dans différents sports.

Néanmoins, pour un club ou une équipe nationale, le maillot est plus qu'un simple vêtement, il est également et surtout le symbole de l'histoire et de l'héritage d'une équipe, 
d'un club, parfois, d'une ville entière. Chez les supporteurs, qui n'a pas rêvé de porter le maillot de football ou l'écharpe de son équipe préférée ? Le maillot de football constitue l'emblème d'une équipe, son signe de ralliement. Manches longues ou courtes, rayés ou unis, ce qui compte c'est que le maillot soit aux bonnes couleurs, celle de son équipe favorite. II est tout d'abord à noter que suivant les pays, les sports collectifs et la tradition, les couleurs de maillot sont différentes.

D'un point de vue historique, à l'époque victorienne en Angleterre, il n'y avait pas d'uniforme ou d'équipement spécifique à porter sur le terrain. Les joueurs exhibaient des maillots blancs de cricket ou tout autre chose qu'ils avaient et les équipes ne pouvaient être distinguées les unes des autres que par des chapeaux de couleurs différentes, des foulards ou des écharpes. Mais, les journalistes et les supporters assistant aux matchs de football ont commencé à exiger que les équipes portent des équipements particuliers pour rendre plus commode la distinction des équipes, des joueurs et des spectateurs. Pour éviter les confusions, le livre des règles du jeu de 1867 recommanda aux joueurs de la même équipe de porter des maillots de couleur similaire. Les tout premiers maillots de football britanniques étaient ornés des couleurs de l'école ou du club sportif à partir desquels l'équipe avait été fondée. Ainsi, le premier maillot Blackburn Rovers était blanc et orné de la Croix Bleue de Malte de l'école de Shrewsbury. Au Royaume-Uni à l'époque, le football était presque exclusivement le sport de la classe moyenne supérieure qui pouvait facilement se permettre d'acheter une chemise aux couleurs de son club. Mais, beaucoup de clubs de la classe ouvrière de l'époque arboraient des chemises blanches, qui étaient bon marché et faciles à trouver. En 1891, lors d'une tournée dans le Wolverhampton, Sunderland portant le même maillot à rayures rouges et blanches que l'hôte, de nouvelles règles furent introduites indiquant que toutes les équipes devaient avoir un jeu de maillots de rechange d'une autre couleur pour éviter de tels incidents (cf. Tunmer \& Fraysse, 1904 ; Pontie, 1905). Au début, c'était l'équipe jouant à domicile qui devait changer de maillot en cas de similitude avec l'équipe visiteuse. Cette règle a été modifiée en 1921 et c'est, maintenant, l'équipe en déplacement qui doit faire le changement. En 1909, afin d'aider les arbitres à identifier le gardien de but, les règles du jeu ont, encore, été modifiées pour indiquer que le gardien de but doit porter un maillot de couleur différente des joueurs de champ de son équipe. Au début, il avait été établi que ces maillots devaient être soit de couleur rouge soit de couleur bleu roi. Quand le vert a été ajouté comme troisième option, en 1912, il est devenu la couleur la plus utilisée. À cette époque, les gardiens portaient généralement une sorte de pull-over qui était plus approprié pour résister au froid que pour la pratique d'un sport. L'ère des équipes avec des jeux de maillots de couleurs distinctes était lancée. En 1953, les joueurs de Bolton 
Wanderers apparurent lors de la finale de Coupe d'Angleterre avec un équipement fait de tissu synthétique brillant. Bien que peu utilisé à l'époque, le maillot de football moderne était né. Mince, léger, ce type de maillot était, pourtant, porté en Espagne et en Italie depuis longtemps. Manches courtes, maillots plus légers sont devenus la norme et les derniers vestiges des équipements de l'époque victorienne ont disparu.

En France, après un début où le football amateur est dominé par l'esprit classique des clubs sportifs réservés à une élite sociale, le football aménage aussi son propre territoire au sein du sport travailliste et dans les clubs populaires. II y apporte ses règles, ses modes de fonctionnement et sa «temporalité décalée', rythmée par les matchs du dimanche et, pour les chanceux, les entraînements en semaine. La distinction travailliste se traduit par les appellations des épreuves, dotées de prix aux noms des journaux ou des figures du mouvement ouvrier mais également par les sigles des clubs (du classique, Prolétarienne Clichoise ou L'espérance Vaillante Granvillaise au poétique, l'Églantine sportive Meudonnaise). Cela passe aussi naturellement par l'héraldique des maillots où, inutile de le préciser, le rouge se décline sous les toutes variantes dans les tenues des joueurs. (cf. Ksiss, 1994).

Ainsi, à première vue, les couleurs brillantes portées par les joueurs de la « Tribu du football » ont l'air d'être simplement décoratives, mais cela est une erreur (cf. Morris, 1981). C'est vrai, elles constituent une touche attrayante, chatoyante au-dessus du vert du gazon mais, en fait, l'intérêt visuel n'est pas la fonction première de ces couleurs. En réalité, elles sont des signaux essentiels pour la tribu. Comme les couleurs exhibées par beaucoup d'animaux ou de fleurs, elles envoient des messages essentiels à propos de ceux qui les portent. En conséquence, cette fonction restreint leur variété. II n'y a aucune équipe, par exemple, jouant avec des maillots couverts de grandes taches roses sur un fond de spirales pourpres. II n'y a pas non plus de clubs qui envoient leurs « héros » sur le terrain en portant des stries grises et marron. Ce qui, au début, a l'air d'être une cacophonie sauvage de couleurs et de dessins, s'avère à l'examen plus proche d'être une gamme sévèrement limitée à peu de choix. En fait, elles autorisent un rapport de complicité infra consciente, ce «sens pratique » qui, selon Bourdieu, permet à chaque individu d'adapter ses comportements dans la société de manière quasi automatique sans recourir à une réflexion consciente et au discours. Elles sont productrices, à l'insu des acteurs eux-mêmes, de tel ou tel registre d'actions ou de représentations. Leur choix ne relève donc ni de l'anodin, ni de l'arbitraire mais répond à un processus de constitution d'une équipe de joueurs en un groupe social clairement identifié susceptible de provoquer l'adhésion / répulsion des supporters dans une sorte de «logique pratique produite en dehors de toute intention consciente » (Bourdieu, 1980, p. 160). 
Une enquête sur les meilleures équipes d'Angleterre, d'Écosse, de France et d'Italie révèle que le dessin des maillots le plus populaire est celui où une seule couleur simple prédomine. Le short et les bas répercutent d'habitude cette couleur ou contrastent avec simplement du noir ou du blanc. La couleur la plus populaire de l'Angleterre et l'Écosse est le bleu. En France et en Italie, on combine souvent deux couleurs mais c'est le rouge qui domine quand-même.

Le dessin de maillot le plus prisé, à part la couleur dominante simple, renvoie aux rayures verticales. Les bandes horizontales de couleur sont aussi appréciées quoique avec modération dans quelques régions et elles semblent complètement absentes de certains pays continentaux. Les autres dessins vus incluent une large écharpe en diagonale, deux couleurs séparées verticalement ou une bande verticale sur le côté à gauche du corps. Les dessins de maillots avec quatre carrés sont des formes plus rares (Figure 1).
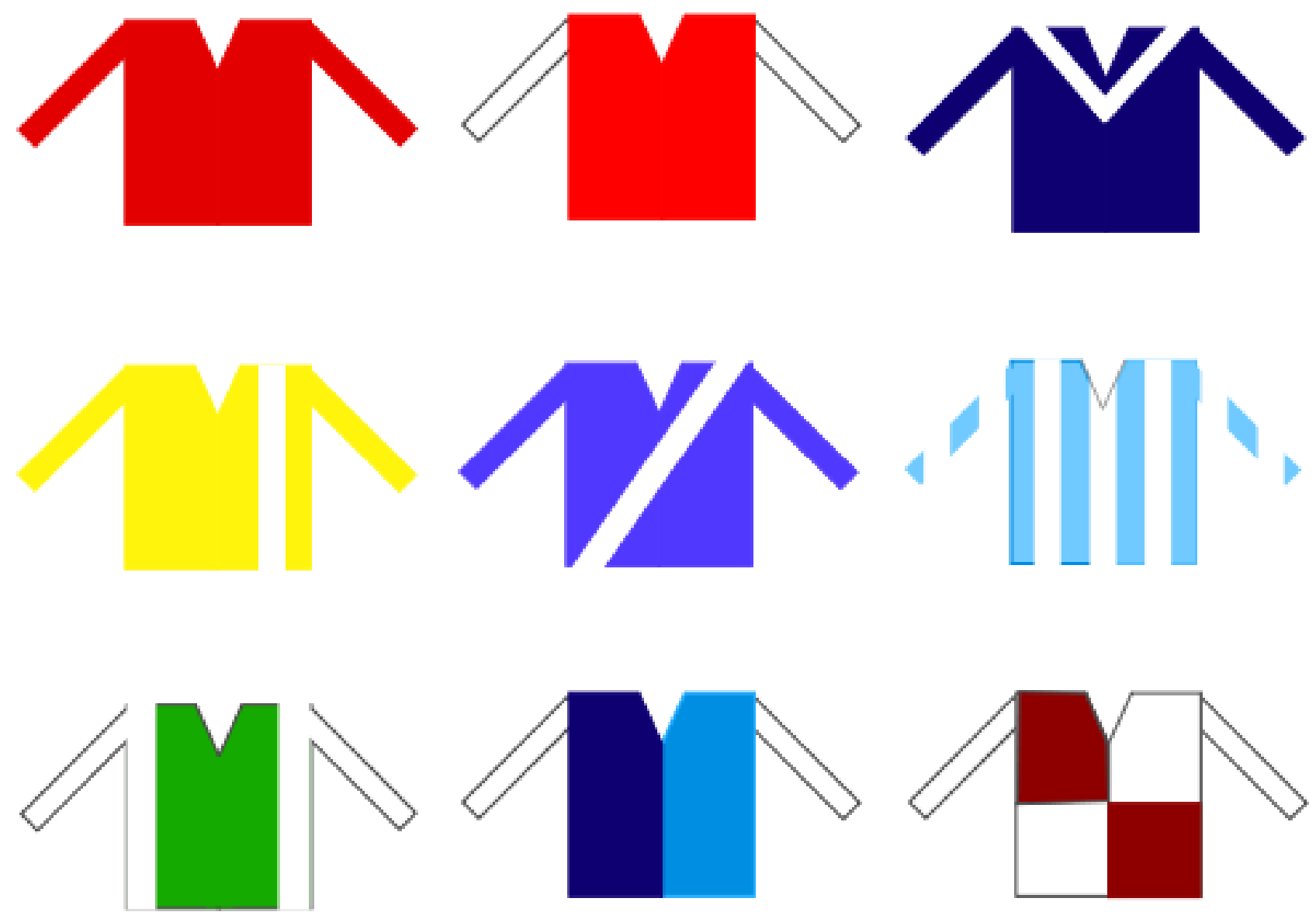

Figure 1. Quelques exemples de dessins classiques sur les maillots.

Pourquoi ces couleurs et ces dessins prédominent-ils ? Pourquoi n'existe-t-il aucun ou peu de dessins avec des fleurs, des pâtés, des taches, des courbes ou des spirales de couleur sur les maillots des héros ? Pourquoi n'existe-t-il quasi aucun maillot multicolore avec d'audacieux dessins ? Pour répondre à ces questions, il est nécessaire d'analyser 
les fonctions essentielles des couleurs dans la Tribu foot qui opèrent comme des signaux visuels fondamentaux.

\section{Les joueurs doivent être bien visibles}

Cela élimine tous les marron ternes, les gris, les délavés ou les pastels. Cela exclut aussi une suite chromatique brouillée, comme celles qui conduiraient à un camouflage des joueurs. De même certaines couleurs brillantes ou dessins audacieux sont inappropriés dans le contexte d'un match de football, à cause des caractéristiques spéciales du jeu. La présence d'une grande balle ronde signifie que d'énormes motifs circulaires sont tabous. II n'y a pas de grandes taches ou des cercles sur les maillots de football (bien qu'ils soient assez communs sur les tuniques de jockeys, dans le monde des courses de chevaux). Le fait que le football soit vu sur un fond vert signifie que même les équipements verts les plus brillants risquent de devenir trop feutrés sur le champ de jeu; en conséquence, le vert est une couleur rare. Arrivé à ce point, on ne sait pas ce qu'en pensent les stéphanois!.

L'exigence la plus importante est que les couleurs, surtout les couleurs de maillots, doivent rendre les joueurs aussi visibles que possible à bonne distance. Pour les joueurs proches, il n'y a aucun problème, même un maillot couvert de boue reste assez visible. Mais quand un joueur fait une longue passe à un coéquipier éloigné, il doit être capable de l'apercevoir, en un clin d'oeil, en un quart de seconde, alors une simple couleur brillante sera, pour le porteur de balle, le meilleur guide. Dans cette optique, un rouge pur est la meilleure de toutes les couleurs. Déjà Mahlo (1969) affirmait que la pratique des jeux sportifs collectifs développe une capacité d'observation permettant de reconnaître des éléments essentiels du point de vue du problème tactique à résoudre. II faut donc travailler ce type d'observation particulier tout en veillant à ne pas seulement augmenter la quantité d'observables à traiter mais aussi à améliorer la qualité des observations faites tout en préservant la rapidité et la sélection de l'essentiel

\section{Les partenaires doivent se différencier des adversaires}

Les pionniers du jeu de football association au dix-neuvième siècle jouaient avec leur propre chemise mais ils furent rapidement confrontés au fait de reconnaître leurs coéquipiers. Il fut donc entériné que chaque équipe se devait d'avoir une couleur distincte. Tant pour les joueurs que les spectateurs, il est, donc, indispensable qu'il ait un contraste entre les couleurs des deux équipes afin de bien suivre l'évolution de l'affrontement. II y a deux façons de répondre à cette règle. Dans le monde de courses de chevaux, chaque propriétaire a un ensemble unique des couleurs pour ses jockeys. Cela donne des 
combinaisons assez compliquées de coloris et de dessins, pour éviter la confusion. Dans le football, il est seulement nécessaire de faire contraste contre un autre jeu de couleurs à la fois. Cela signifie que si chaque équipe porte toujours ses propres couleurs à domicile, elle possède un deuxième jeu de maillots avec d'autres couleurs pouvant être porté à l'extérieur ; comme cela, il n'y a aucun problème. Beaucoup de matchs ne nécessitent pas de changer les couleurs familières, mais s'il y a un problème, on a recourt au deuxième jeu de maillots.

Pour des raisons psychologiques, les joueurs préfèrent jouer dans leurs couleurs habituelles et ne sont pas très heureux quand ils doivent porter leur deuxième jeu de maillots pour les matchs à l'extérieur. Les supporteurs qui les suivent et qui portent des écharpes ou agitent des drapeaux aux couleurs habituelles, préfèrent aussi voir leurs héros dans leurs costumes familiers. Ainsi, il y a une certaine pression pour éviter de faire trop de changements. Cela a pour effet de garder les équipements aussi simples que possible. Si, par exemple, un club donné avait un maillot éclatant rouge, jaune et bleu, cela entraînerait un changement de maillot contre les équipes dont le maillot habituel contient du rouge, du jaune ou du bleu. Cela couvrirait la majorité des équipes rivales et exigerait du club de jouer à l'extérieur presque toujours avec son deuxième jeu de maillots. Si les couleurs habituelles sont une couleur simple, dominante, disons, bleu, il y a seulement obligation de changer en visitant un "club bleu". C'est pourquoi, les équipements d'une couleur sont les plus populaires et les plus nombreux ; c'est une raison supplémentaire qui explique pourquoi les teintes compliquées sont extrêmement rares. Les avantages d'avoir un maillot unique, bien identifiable, sont confortés par le désir de conserver ses couleurs habituelles pour le plus grand nombre possible de matchs à l'extérieur.

Attrill, Gresty, Hill \& Barton (2008) ont montré que depuis 1947, les équipes de football anglaises vêtues de maillots rouges ont été champions plus souvent que prévu par rapport à la proportion des clubs jouant en rouge. Pour vérifier si cela indique une amélioration de la performance à long terme au sein d'équipes portant du rouge, nous avons analysé les positions des équipes de la ligue professionnelle de football en fonction des différentes teintes portées. Dans toutes les divisions de la ligue, les équipes rouges ont un meilleur rendement à domicile, avec des différences significatives tant en pourcentage du maximum de points obtenus et leurs positions moyennes dans le classement de la ligue. Aucune différence significative n'a été observée pour des matchs à l'extérieur, quand, bien souvent, les équipes ne portaient pas leurs couleurs habituelles. Une analyse appariée par paire de rouge et de non - rouge portant sur huit villes anglaises montre de bien meilleures performances pour les équipes en rouge sur une 
période de cinquante-cinq ans. Enfin, les effets sur la réussite à long terme pourraient avoir des conséquences pour la sélection des couleurs dans les sports d'équipe ; le port du rouge améliore les performances dans une grande variété de contextes concurrentiels et devrait donner un nouvel élan pour l'étude des mécanismes sous-jacents à ces effets. Hill et Barton (2005) ont proposé une explication au fait que les sportifs qui portent du rouge gagnent plus souvent: cela pourrait refléter un signal de position dominante comme dans une grande variété d'espèces animales.

Nous allons y revenir plus précisément dans un prochain paragraphe.

\section{Les supporters doivent se différencier de leurs proches voisins}

Contrairement à la France, en Angleterre il y a souvent deux voire plusieurs clubs dans une ville ou un comté. Qu'une grande agglomération ait deux clubs de football importants et il doit y avoir un contraste chromatique important dans les maillots que portent les joueurs, pour des raisons de définition de territoires. Les fans des deux clubs veulent être capables de se distinguer l'un de l'autre, plus particulièrement, lors du derby annuel, quand les deux clubs locaux et rivaux se rencontrent. À ce moment, chacun doit pouvoir porter, fièrement, les couleurs de son champion. Ainsi, Manchester United est en rouge et Manchester City est en bleu; Liverpool est en rouge et Everton est bleu, Nottingham Forest est en rouge et Notts County est en noir et blanc ; etc. Avec la capitale, cela devient un vrai problème, en effet à Londres, il y a douze clubs de Ligue professionnels et cela étend le spectre des couleurs au maximum. Milan AC et inter de Milan, Real de Madrid et Athletico de Madrid en sont d'autres exemples patents en football.

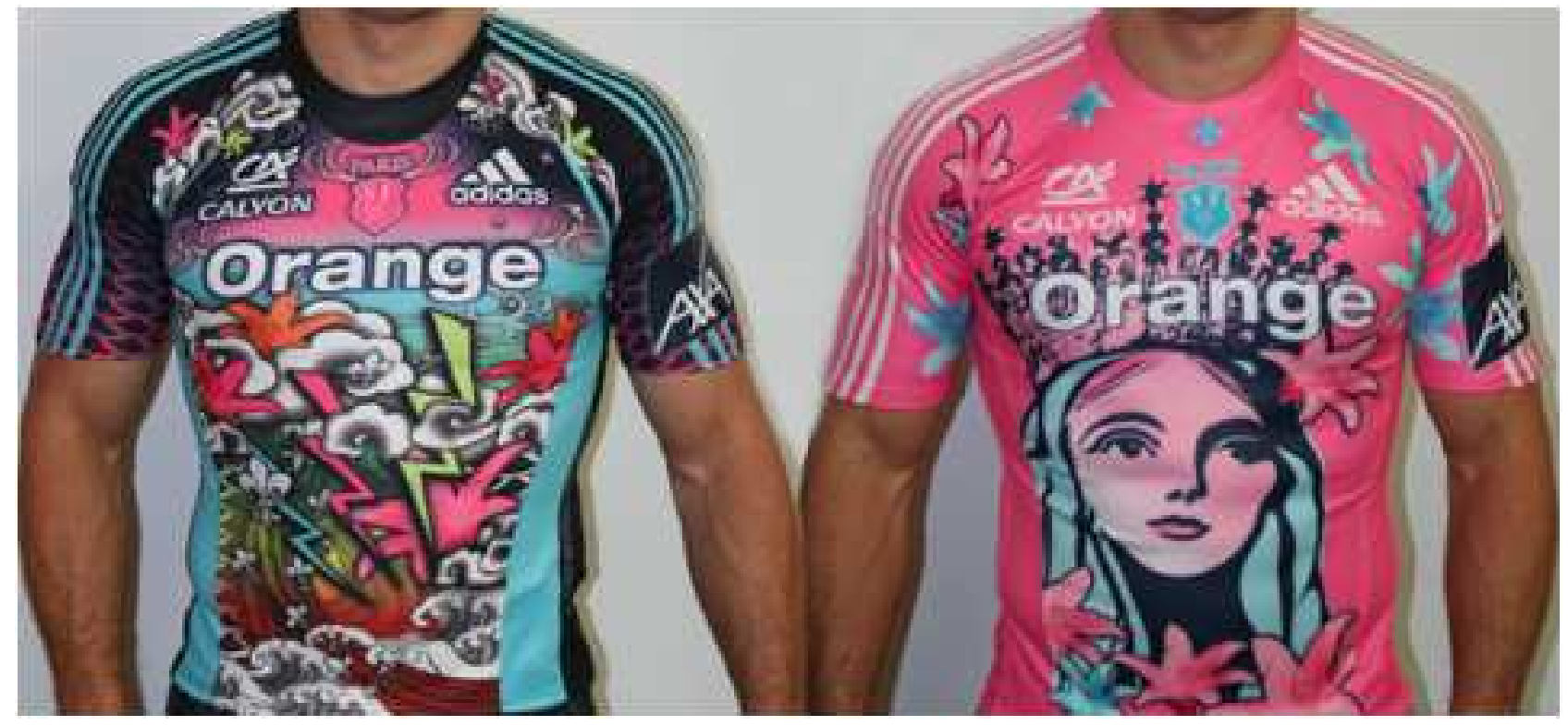

Figure 2. Maillot pour la saison 2009 - 2010 du Stade Français. 
Racing Métro 92 et le Stade Français illustrent, aussi, ce fait en rugby. Ce dernier club, ultime provocation arbore parfois depuis 2005, un maillot et un short rose, couleur considérée comme aux antipodes de la virilité affichée des rugbymen (Fig. 2). Ce maillot a été endossé par les joueurs de l'équipe fanion du club pour la première fois lors d'un match de championnat à Perpignan. Malgré la défaite sportive de ce jour, le Stade français vendra cette année-là 20000 exemplaires de ce maillot.

\section{Les maillots doivent donner un avantage psychologique à ceux qui les portent}

Les couleurs extrêmement voyantes ne sont pas simplement faciles à voir pour passer la balle à un coéquipier, elles sont aussi intimidantes pour l'ennemi. Au niveau non conscient, elles envoient un message disant, "je suis courageux", "je ne suis pas effrayé par toi". Plus brillante est la couleur, plus féroce semble son propriétaire. À cet égard les footballeurs ressemblent aux animaux assez dangereux. N'importe quelle espèce d'animal qui possède une arme défensive spéciale, comme le serpent avec son venin ou la guêpe avec son dard annonce ce fait à ses ennemis potentiels. Il le fait en portant une « couleur qui effraie » en quelque sorte, à laquelle les ennemis réagiront aussitôt qu'ils la verront. Les serpents venimeux ont souvent des bandes brillantes d'orange et noir, les guêpes sont brusquement marquées dans jaune et noir. Presque tous les animaux venimeux ou dangereux annoncent leur présence avec leurs couleurs et il est surprenant de constater que l'on peut faire le parallèle avec les tenues de football, surtout avec les dessins contenant des stries ou des bandes. L'avantage de porter des pièces de couleur très contrastées consiste en ce que ces lignes tranchées restent, même si le fond change. Et cela s'applique, aussi bien aux animaux qu'aux footballeurs (Morris, 1981).

On peut avancer comme explication que les «couleurs d'animaux venimeux » donnent aux adversaires une sensation non consciente qu'ils peuvent être "piqués" ou "empoisonnés" par un contact trop proche. Même si cet avantage psychologique opère à un niveau infra conscient, il ne doit pas être ignoré. Les joueurs eux-mêmes peuvent rire d'une telle suggestion, mais il n'est pas facile d'être certain de l'impact, effectif, de différentes couleurs quand elles sont portées par un joueur qui avance vers vous à toute allure. II y a une forte chance que, si le joueur est habillé dans des couleurs éclatantes agressives et contrastées, comme un insecte piquant géant, il semblera plus menaçant que s'il est habillé avec un bleu pâle ou un coloris pastel.

Ce fait établi, il pourrait sembler logique de s'attendre à la prédominance du rouge, du jaune ou de l'orange parmi les « couleurs de la Tribu » au lieu du rouge, du bleu et du blanc. Bleu, même bleu foncé, semble manquer de férocité pour une couleur si populaire. Blanc semble délavé et incolore. Les jaunes et l'orange, sont si répandus parmi les 
animaux dangereux qu'il est curieux de ne pas les trouver dans de plus grandes proportions. Quelle est l'explication de ces différences?

II semble que ces associations de couleurs entrent en conflit avec d'autres valeurs. Jaune, bien que ce soit une couleur joyeuse, radieuse représentant le soleil et le sable, est aussi une couleur associée à la lâcheté et à la quarantaine. L'expression "c'est un jaune" est bien connue et largement utilisée comme insulte ce qui a beaucoup joué contre la popularité de cette couleur. La couleur orange pourrait sembler échapper à cette étiquette nuisible, mais elle souffre d'un autre désavantage. C'est une couleur intermédiaire - à mi-chemin entre jaune et rouge. Tout l'intermédiaire colore peu les maillots dans la Tribu du Football, ainsi que toutes les versions pâles de fortes couleurs (comme le rouge délavé, le bleu pâle, etc.). Une couleur intermédiaire a l'inconvénient psychologique de représenter "l'indécision" : il a du mal à se décider. Orange est-il un rouge jaunâtre ou un jaune rougeâtre ? Cela nuit à la netteté de son image et, par conséquent, le réduit à une couleur relativement rare dans la Tribu.

Si l'on regarde les couleurs les plus répandues, le rouge est facile à comprendre. C'est la plus notable de toutes les couleurs, surtout à distance et elle a un impact symbolique puissant comme couleur du sang, représentant ainsi énergie, vie, force, pouvoir et intensité. C'est la couleur parfaite pour n'importe quelle équipe sportive et il est difficile de comprendre pourquoi, dans quelques pays, elle n'est pas encore plus populaire que cela. Le grand attrait du bleu est plus dur à expliquer. En beaucoup de choses, c'est l'opposé du rouge, c'est la couleur de la paix, du repos, de l'harmonie et de la loyauté. Peut-être son secret en est la direction de son message. Peut-être que son attraction la plus grande est constituée par les signaux calmants qu'il envoie d'un coéquipier à l'autre, plutôt que le message agressif transmis par les maillots rouges vers leurs adversaires (Morris, 1981).

Le blanc est une autre couleur (ou plutôt, pour être techniquement exact, un manque de couleur) dont la popularité est difficile à comprendre. Symboliquement, blanc est la couleur de la mort et de la peur, la glace, la neige et le froid ainsi que la pureté et l'innocence et ne symbolise pas les qualités les plus nécessaires dans le monde sportif. En plus, c'est vite sale et paraît crasseux pendant tout le match. Et pourtant le blanc reste une des trois couleurs les plus privilégiées. La seule explication de sa popularité (à part le fait d'attirer l'attention) est qu'il représente vraiment un autre symbole, à savoir celui du héros. Du temps où beaucoup de clubs devenaient pérennes, à la fin du dix-neuvième siècle ou du début du vingtième, il y avait deux expressions d'usage commun : il est « blanc comme neige » et « le côté noir des choses » de l'autre. Dans ce contexte, blanc signifie honorable ou équitable, pendant que noir signifie infâme ou sinistre. La distinction était fondée à l'origine sur une vue raciste de suprématie blanche sur les sauvages noirs 
et qui a survécu dans le symbolisme chromatique même là où le racisme a, en partie, disparu. Nous parlons toujours de "jours noirs" avec comme sens, jours mauvais. Par contraste, blanc devient la couleur de la valeur, de l'honneur et de l'héroïsme. C'est de cette manière, sans doute, qu'il a survécu comme une des trois premières couleurs du monde du football.
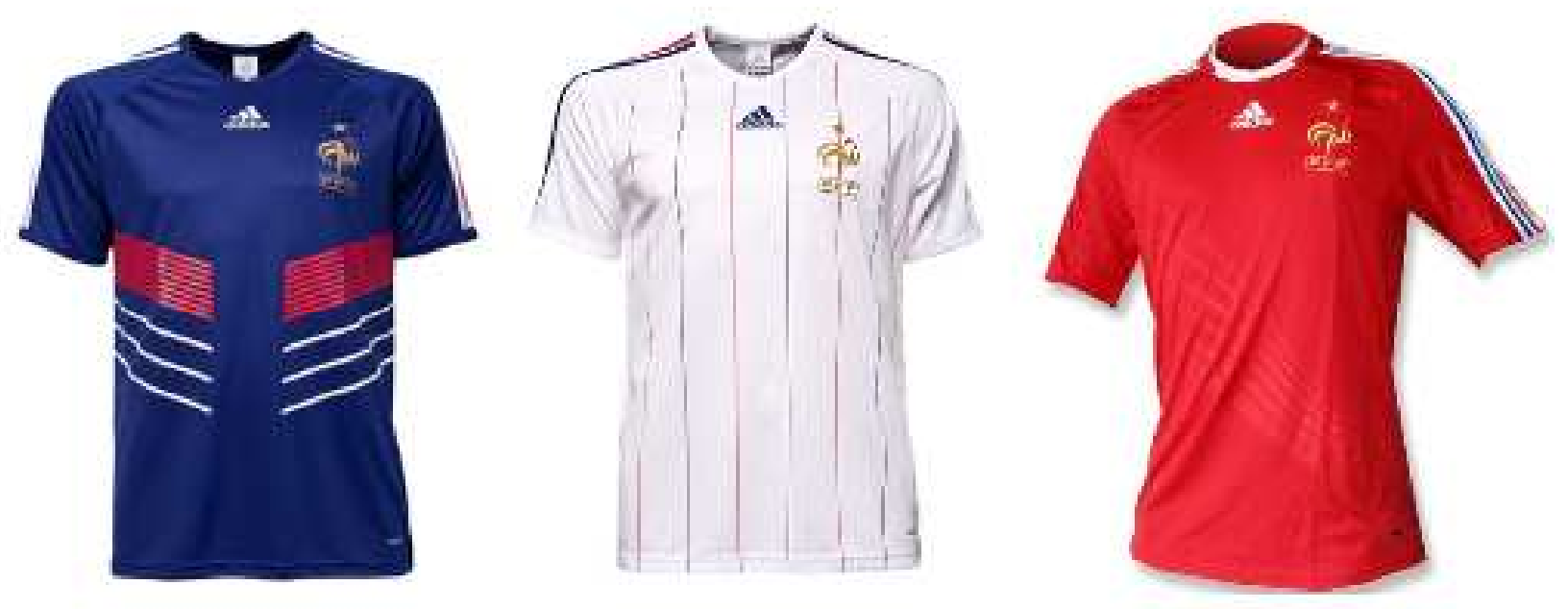

Figure 3. Jeu de maillots Adidas de l'équipe de France pour la Coupe du Monde 2010.

Quant à elle, l'équipe de France de football suivant les circonstances jouent en bleu, en blanc ou en rouge (cf. Fig. 3). Hubac (2004) précise que pour son premier match, le 1er mai 1904 face à la Belgique, l'équipe de France portait un maillot blanc avec, en écusson, les deux anneaux rouge et bleu entrelacés de l'Union des sociétés françaises de sports athlétiques. Le 1er novembre 1906, pour le premier France - Angleterre de l'histoire (et une défaite 15-0), la France évoluait avec un maillot rouge. II est à noter que c'est la première utilisation d'un deuxième jeu de maillots dit de rechange (les Anglais portant aussi un maillot blanc...). Ce même rechange passant du rouge au bleu à partir de la rencontre Angleterre - France (défaite 12-0) du 23 mars 1908, premier match de l'équipe de France avec un maillot bleu, un short blanc et des bas rouges... Un maillot blanc rayé de bleu verticalement avec un col rouge est employé jusqu'à la première guerre mondiale, de 1910 à 1914. Ensuite le maillot bleu s'imposera définitivement comme première tenue. Dans les autres pays également, après le rouge, le bleu et le blanc, les autres couleurs les plus privilégiées sont le jaune et l'orange, bien que ceux-ci ne soient nulle part particulièrement communs. Les couleurs rarement utilisées incluent le vert, le bordeaux, et le noir, le bleu pâle ou le violet. Les roses bonbons, les gris et les marrons sont pratiquement non existants en football. 
Pourtant, le maillot jaune et le short vert du Brésil sont, sans doute, l'assortiment de couleurs le plus connu à l'heure actuelle, mais ce maillot n'a pourtant pas toujours été si caractéristique. En effet, l'équipe du Brésil a porté longtemps un maillot blanc jusqu'à sa défaite face à l'Uruguay en 1950. Cette finale de la Coupe du monde de football qui était organisée à domicile, vit l'Uruguay battre le Brésil. Cela fut vécu comme un véritable drame national et marqua la mémoire des 199854 personnes présentes pour l'occasion. Le Brésil, qui n'avait besoin que d'un match nul pour devenir champion du monde, et qui menait 1-0, fut finalement battu 2-1, par l'équipe d'Uruguay. Cette profonde déconvenue a rappelé à l'ensemble du peuple brésilien traumatisé et superstitieux que le blanc était souvent associé au deuil et à la peine. Les dirigeants brésiliens ont donc décidé d'adopter les couleurs verte et jaune du drapeau national, qui symbolisent les forêts de l'Amazonie et les réserves d'or du pays. C'est donc avec ces couleurs que le Brésil gagnera cinq Coupes du Monde...

Une autre tendance très répandue et sans aucun attrait a été l'ajout de publicités sur les maillots des joueurs. Les compagnies importantes sont disposées à payer de grosses sommes pour sponsoriser des clubs et faire écrire leurs noms en travers des larges poitrines des héros locaux. Cela est peut-être une proposition commerciale intéressante, mais c'est aussi une attaque de l'image des joueurs, en les réduisant à la condition d'hommes sandwich et en les privant d'une grande partie de leur dignité. Néanmoins, aujourd'hui, même le plus petit des clubs amateurs arbore une marque sur son maillot. Très récemment, les derniers résistants à cet état de fait ont cédé. Le FC Barcelone et l'Atletico Bilbao avaient durant plus d'un siècle fièrement porté un maillot vierge de toute publicité. Le train de vie du football business a mis fin à ces belles idées. Pour l'instant, la « réclame » n'a pas atteint les maillots des équipes nationales mais dans d'autres sports collectifs, moins puissants économiquement il est vrai, les équipes nationales affichent pour la plupart une marque.

\section{Discussion}

Mais, les choses évoluent aussi concernant les équipes de club. Par exemple, pour une rencontre $\mathrm{OM}$ - Milan $\mathrm{AC}$, qu'espère-t-on d'un tel match au-delà du résultat de la rencontre ? On s'attend à une confrontation de deux clubs importants, chargés d'histoire et de tradition, fiers de leurs couleurs ! On s'attend à voir se confronter les blanc et bleu contre les rouge et noir comme lors des grandes soirées européennes ! Eh bien, hier soir, contre toute logique Marseille jouait en noir et Milan en blanc. Cela n'est pas une première... Souvenez-vous du Marseille-Liverpool de l'année dernière (Marseille en jaune, Liverpool en noir) ou encore de l'insolite Espagne - France d'avant l'Euro 2008. Ce 
soir-là, les Bleus arboraient un nouveau maillot rouge, ce maillot rouge étant le second maillot de l'équipe nationale française. Comme le rouge est la couleur traditionnelle du maillot de l'Espagne, les Espagnols ont étrenné ce soir-là, leurs nouveaux maillots prévus pour les matchs à l'extérieur, un maillot de couleurs jaune et ocre.

Alors évidemment c'est du marketing... L'objectif ici est de montrer au public la nouvelle collection de maillots pour susciter l'envie d'acheter De façon évidente, cela n'a aucune influence sur la vie du club et bien sûr, le poids de la tradition ne doit pas nous empêcher d'oser de nouvelles choses ; non mais !

Le procès qui est fait ici est celui des équipementiers et des dirigeants qui adoptent une politique commerciale visant à proposer un maximum de produits dérivés aux supporters. Si le maillot ne changeait jamais, quel intérêt aurait un supporter à en acheter un nouveau chaque année ? Donc à part refaire le procès du marketing, il n'y a pas grand-chose à ajouter. Mais, ce qui est condamnable, aussi, c'est l'attitude des clubs ou des fédérations qui laissent faire. Bien sûr, un équipementier qui parraine un club ou une sélection pense avoir des droits. Mais a-t-on déjà vu l'équipe nationale anglaise arborer un maillot autre que blanc à domicile ? Et le Brésil, vous imaginez maintenant les joueurs au stade Maracaña de Rio sans leurs couleurs jaune et verte, mais en violet ou en fuschia. Là aussi, de façon évidente, pour certains clubs ou certaines sélections, le respect des couleurs est un élément non négociable du contrat, un élément intangible du cahier des charges. Cet ensemble témoigne que l'identification à un club ne passe pas uniquement par des résultats et des joueurs, mais aussi par un stade et... des couleurs.

Pour en revenir à OM - Milan AC, c'est lorsque que les 22 joueurs ont échangé leurs maillots à la fin du match que les codes ont été, de nouveau, respectés. D'ailleurs, il est à noter que cette tradition d'échange de maillots date du 14 mai 1931. Ce jour-là, la France battit l'Angleterre 5 buts à 2. À la fin de la rencontre, afin de garder un souvenir de cette mémorable victoire, les joueurs français proposèrent aux joueurs anglais d'échanger leurs maillots et ceux-ci acceptèrent. Pour clore cette petite histoire, les tenues françaises étaient en coton, et les maillots des Anglais étaient des chemisettes en soie d'où le nom « shirt » pour un maillot en anglais alors qu'une traduction littérale serait plus proche de «jersey ».

S'intéresser ainsi aux histoires et légendes des maillots pourrait au premier abord sembler anecdotique, voire triviale, peu importe après tout que les joueurs endossent des maillots rayés ou unis, rouges ou verts. Ce serait toutefois oublier leur fonction emblématique et identitaire. Avec eux, le corps se fait signe et se charge de sens. Il devient le support de représentations et de pratiques particulières qui permettent de délimiter des territoires et de se différencier. Finalement, le maillot exacerbe la puissance sémique du corps. 
L'intérêt et l'attention que les responsables sportifs accordent au choix de cette parure sont révélateurs de sa force symbolique mais aussi pratique. Sur le terrain, il rend visible, façonne l'identité, marque des appartenances, participe de la fabrication d'un ascendant psychologique dans le rapport d'opposition, bref il oriente la pratique elle-même. II transforme le corps en «instrument et espace de communication et de signification » dans la mesure où il donne à lire à autrui « les signes et les symboles d'un lignage, d'un rang, d'une condition, d'un engagement » (Berthelot, 1998). Cet usage sémiologique du corps ne se déploie pas uniquement sur les stades de football mais trouve son expression dans d'autres pratiques contemporaines (par exemple le tatouage, la mode dans le prêt à porter). Comme le souligne Berthelot (1998), «l'infinie diversité des formes sociales de mise en jeu du corps humain semble défier l'analyse ». Le choix des maillots en offre une illustration remarquable.

Enfin, en rêvant beaucoup, on pourrait penser que porter le maillot national est un honneur et ne devrait procurer d'autre rémunération que la fierté de représenter son pays sur la scène internationale. Les sommes générées pourraient, par exemple, être entièrement reversées au sport pour tous et au sport amateur qui en ont tellement besoin. En effet, aucun joueur n'est obligé d'accepter de toucher un cachet pour sa sélection à une Coupe du monde. Le seul fait d'y participer augmente, de facto, sa "valeur" sur le marché des transferts et le place déjà dans de bonnes conditions pour négocier ses contrats...

\section{Bibliographie}

Attrill, M.J., Gresty, K.A., Hill, R.A., \& Barton, R.A. (2008). Red shirt colour is associated with long-term team success in English football. Journal of Sports Sciences, Volume $26(6), 577$ - 582.

Berthelot, JM. (1998). Le corps contemporain : figures et structures de la corporéité. Recherches sociologiques, 1998/1, 7-18.

Bourdieu, P. (1980). Le sens pratique. Paris : Editions de minuit.

Hill, R.A., \& Barton, R.A. (2005). Red enhances human performance in contexts. Natures, 435, 293.

Hubac, T. (2004). 1904-2004. Un siècle en Bleu. Paris : Mango Sport.

Ksiss, N. (1994). Les Origines du sport ouvrier en Europe, Paris, L'Harmattan.

Morris, D. (1981). The soccer tribe . London : Jonathan Cape.

Pontie, E. (1905). Le football association. Paris : Lucien Laveur.

Tunmer, A.A., \& Fraysse, E. (1904). Football (Association). Paris : Colin. 